\title{
17
}

\section{MOSQUITO UTOPIAS AND DYSTOPIAS}

\section{A dispatch from the front lines}

\author{
Indra Vythilingam
}

Utopia is a term to describe things that are perfect in all aspects whereas a dystopia is just the opposite; both share characteristics of science fiction and fantasy. But when considering mosquitoes, one should bear in mind that these creatures have lived for aeons on planet Earth and they too need to survive. As mentioned in earlier chapters there are about 3,500 species of mosquitoes but only a few hundreds of them are disease vectors. They carry either viruses or parasites that can infect humans and animals and which can lead to mortality if not treated. However, to a lay person, a mosquito is a mosquito, and is responsible for the misery it causes.

Control measures are instituted only towards mosquitoes that are vectors. This is especially true in tropical countries which are burdened with vector-borne diseases. The control measures are targeted to the behaviour of the mosquitoes and thus, only some non-vector mosquitoes and insects will be affected by these measures. The World Health Organization promotes elimination of diseases like filariasis and malaria (WHO 2017) and many countries have obtained elimination status. But this does not mean that the vectors have all been eliminated. Rather, we have achieved "anophelism without malaria": the requisite vectors are still there but without the pathogens they used to carry. And in the case of malaria as well as other vector-borne diseases, it is only the female mosquitoes feeding on blood that transmit the disease.

How do mosquitoes behave in the human community? What are the forces which determine whether the diseases carried by them will sweep through, leaving a trail of death and disaster; or entrench themselves for a long-drawn struggle; or invade but lightly and disappear? There is no simple answer to this complex situation. Vector-borne diseases in a community are social expressions of the biological relationship between the pathogens, their human hosts and the mosquitoes which bring all of them together. The different species of mosquitoes 
responsible for these different diseases have developed their roles very well and each species has its own niche as described in the early chapters of this book. We know that Ae. aegypti and to some extent Ae. albopictus are responsible for the spread of many arboviral diseases such as dengue (Solomon 2006). Some species of Anopheles are responsible for the spread of malaria while Culex quinqefasciatus and species of Mansonia are responsible for the transmission of filariasis (Wharton 1960). Unfortunately, Southeast Asia has the greatest number of Anopheles species that are vectors of malaria (Hii and Rueda 2013).

Here I summarize the various control measures that have been instituted against the vector mosquitoes and the pros and cons of the new control measures to be instituted. It is very clear that countries plagued by vector-borne diseases are only targeting the vector mosquitoes. All other mosquitoes on this planet can survive.

\section{Anopheles and malaria}

Although there has been great progress in the reduction of malaria infections over the years, the number of malaria cases worldwide in 2018 was about 228 million, of which $93 \%$ occurred in the African region, followed by $3.4 \%$ in Southeast Asia (WHO 2019). Fortunately, there are reasonably effective drugs to control malaria and so some might raise the question why should one even try to kill the mosquitoes? An argument in point is that the threat of malaria is not shared equally across the population: pregnant women and children are most susceptible to the disease, with high risk of mortality if not treated early enough. It is therefore of crucial importance to control these vector mosquitoes to prevent the transmission of the parasites to humans.

\section{Control of Anopheles larvae}

The history of malaria control in Malaya must be attributed to Sir Malcolm Watson who started his work here in Klang in 1900 (Watson 1921, Singh et al. 1988). As a district surgeon he was seeing many malaria cases in a district hospital, as in 1901 when there was a huge epidemic rampant in that region. Watson was attracted to the idea of trying to prevent malaria rather than merely treating cases admitted to the hospital. He initiated a programme of clearing and drainage of the foothill swamps in and around the town. They used both the herringbone drains and the foothill contour drains which proved to be effective (Watson 1921). The number of malaria cases fell from more than 300 in 1901 to 50 in 1903.

At about the same time in Port Swettenham (near Klang), which was opened in 1901, about $68 \%$ of the labourers and government staff came down with malaria. The species that was responsible for malaria in the area was An. sundaicus which was breeding in brackish water. Thus, bunding (or diking), drainage and the exclusion of saltwater by building tide-gates were carried out. These 
measures proved to be successful in the port area (Figure 17.1). Subsoil drains were also built in other areas around Kuala Lumpur where An. maculatus was the vector (Singh et al. 1988). These mosquitoes breed in streams open to sunlight.

Anti-malaria oil was also applied to all breeding sites. This was discontinued when oil became expensive and the insecticide Abate $500 \mathrm{E}$ was introduced. However, over time when these drains with clean water became sullage drains as people settled in these areas, larviciding was stopped since Anopheles breed in clean water and not in sullage (or dirty) water. As Watson described his work controlling mosquitoes: "As we learn more, perhaps the time will come when we shall be able to say to one species of Anopheles, 'Come,' and to another, 'Go,' and shall be able to abolish malaria with great ease, perhaps at hardly an expense" ( (Watson 1921: 292) (Figure 17.1).

\section{Control of Anopheles mosquitoes}

DDT has been used for control of malaria vectors with success as mentioned in several chapters of this book. The publication of Rachel Carson's Silent Spring caused a hue and cry against the use of DDT, especially for agricultural purposes. However, for malaria control it would still be allowed on a case-by-case basis (Mouchet 1994). In Malaysia, a pilot project was started in 1960 in the coastal area of Selangor to determine the efficacy of DDT to control malaria. There were 33 Anopheles species in the area and of these only five were known to be vectors (Moorhouse 1965). DDT was sprayed at $2 \mathrm{gm} / \mathrm{m}^{2}$ on the inside walls

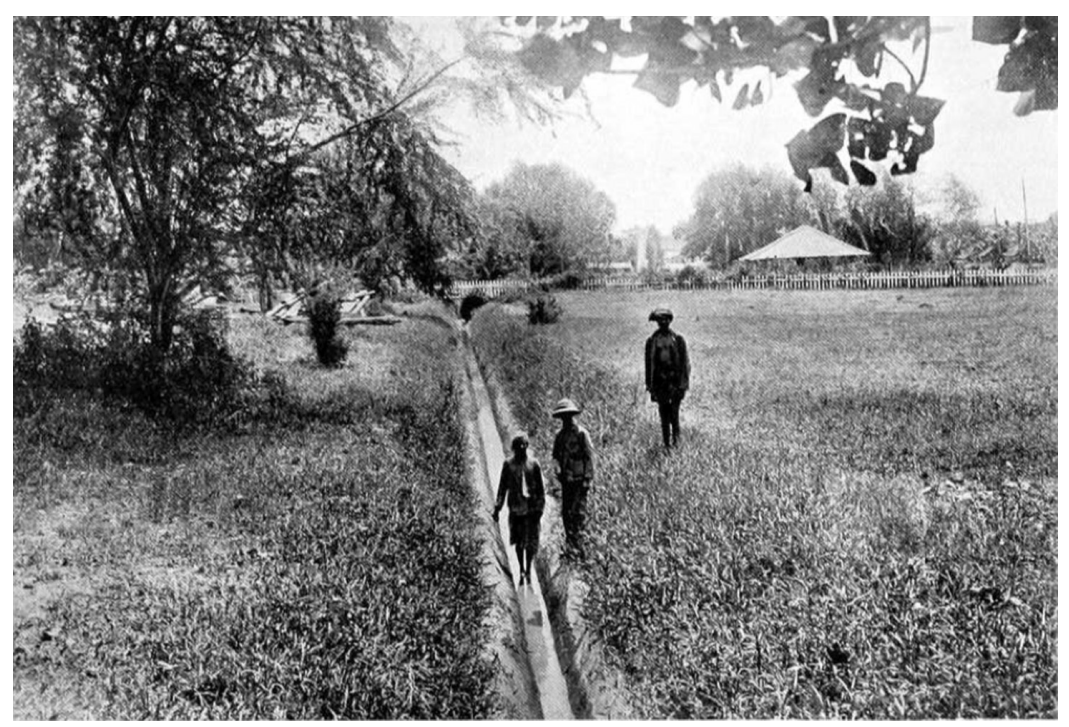

FIGURE 17.1 Inspecting a bunding near Klang. From Malcolm Watson 1921. The prevention of malaria in the Federated Malay States: a record of 20 years' progress. E.P. Dutton \& Company. 
of houses-referred to as indoor residual spraying (IRS). This was carried out because it was observed that the Anopheles mosquitoes, after entering the house, rest on the wall, bite a human and then rest on the wall again before flying out. Thus, Anopheles mosquitoes are able to absorb enough insecticide to kill themselves. The vectors were An. campestris, An. letifer, An. maculatus, An. sundaicus (now known as $A n$. epiroticus) and $A n$. umbrosus. The house-spraying apparently eradicated the An. campestris species but not the rest (Moorhouse 1965). This is mainly due to their different behaviour patterns.

With the introduction of DDT to malaria control and, of course, detection and treatment of human cases, malaria was considerably reduced in coastal areas but the results were less noticeable in the hilly areas of Malaysia. In the late 1980s it was observed that the Anopheles mosquitoes began entering houses to bite and then exit without resting on the walls. This was when insecticide-treated bed nets (ITNs) were tested and found to be useful. It has been reported that a large percentage of malaria has been reduced due to the use of ITNs (Fegan et al. 2007).

However, the problem now faced by the malaria programme is that the vectors are biting in the early part of the night and more commonly outdoors rather than indoors. Previously the peak biting times would be around 10.00-12.00 pm but now it is $7.00-8.00 \mathrm{pm}$. It should be noted that the control is targeted towards the vector; if other pest mosquitoes show similar behaviour, of course there is a possibility that they will be killed.

\section{Monkey malaria infecting humans}

Besides the main pathogens of human malaria, the simian malaria parasite of Plasmodium knowlesi is now infecting humans. All countries in Southeast Asia have reported cases of $P$. knowlesi infections, with the exception of Timor-Leste (Vythilingam et al. 2018). The Leucosphyrus group of Anopheles mosquitoes, which feeds on the long-tailed macaques, becomes infected by biting these monkeys and then transmitting the parasite to humans who they bite later. The first such case was reported in 1965 in Malaysia (Chin et al. 1965) and then a second case in the 1970s (Fong et al. 1971). After extensive research, it was postulated that simian malaria will remain in monkeys and will not be transmitted to humans (Warren et al. 1970). But then a large outbreak of P. knowlesi was reported in 2004 (Singh et al. 2004) to now become the predominant species affecting humans in Malaysia (Hussin et al. 2020). Moreover, a second simian malaria parasite, P. cynomolgi, is now being transmitted to humans in Southeast Asia (Ta et al. 2014, Singh et al. 2018, Grignard et al. 2019, Imwong et al. 2019).

The reason for the transmission of these two malaria parasites is perhaps due to deforestation and other human changes to the landscape (Fornace et al. 2019). Long-tailed macaques are now found on the forest fringe, adjacent to new farms, so that mosquitoes that were originally only forest-dwelling, have followed macaques to their new surroundings (Vythilingam et al. 2018). In the 1960s, 
mosquito species that were mainly biting macaques (Warren and Wharton 1963) are now biting both macaques and humans (Vythilingam et al. 2018). These mosquitoes are biting in the early part of the night and outdoors (Vythilingam et al. 2018), making control measures difficult to conduct since it is not possible to treat the macaques. Control of mosquitoes is especially difficult in the forest and forest fringes. This is how new diseases emerge all the time.

\section{Aedes and arboviral diseases}

Arboviruses transmitted by the Aedes mosquitoes (Ae. aegypti and Ae. albopictus) are mainly chikungunya, dengue and Zika. The history and natural history of each of these viruses have been well elaborated in various chapters of this book. Here I mainly add how other mosquitoes and insects can be affected by some indiscriminate methods used for controlling vector mosquitoes. For example, dengue became a problem in Southeast Asia in the early 1970s, but since there are no drugs to treat dengue, vector control became the hallmark for controlling this disease in the region.

\section{House-to-house larval surveys}

House-to-house larval surveys were conducted to compute the House Index and the Breteau Index, which are measures to determine the density of mosquitoes in an area. In most urban areas in the early 1970s and 1980s, water supply was a problem, so people used to store water, sometimes in open containers. As a result, the Ae. aegypti index was very high. With continuous house surveys and health education, the Aedes House Index in Malaysia has been reduced from 58.8\% to 2.0\% (Mudin 2015). Nonetheless, dengue cases have increased over the years. In Malaysia and in Singapore, the enactment of a new law, "The Destruction of Disease-Bearing Insects Act (DDBIA)," meant that people could be penalized if mosquitoes were found breeding in their homes (Ooi et al. 2006, Vythilingam and Wan-Yusoff 2017). People are now advised to apply the larvicide temephos (Abate $1 \mathrm{SG}$ ) to their storage water or else ensure that water containers are well covered to prevent mosquitoes from laying eggs there. In Vietnam, biological control with the crustacean, Copepod mesocyclops, has been used for feeding on the Aedes larvae (Nam et al. 2012).

\section{Adult control}

Fogging or ultra-low volume (ULV) insecticide spraying, is only carried out when dengue cases are reported, and this is done to kill infected adult mosquitoes to break the chain of transmission. All other mosquitoes and insects will be killed as well. Fogging is carried out at the house where the infection case occurred and in houses within 200 metres. However, during an epidemic, ULV spraying is conducted on a large scale since it can cover larger area. Studies have shown 
that ULV spraying is not very effective since the droplets only get carried as far as the living room of the house and thus mosquitoes hiding in closets and bedrooms will not be killed (Vythilingam and Wan-Yusoff 2017).

It was also found that there was no significant difference when ovitraps were set before fogging, during fogging and after fogging, as the first pupae emerged around day ten on all occasions (Chua et al. 2005). There was also no significant difference in the number of immature Aedes mosquitoes during the three fogging periods (Chua et al. 2005). It shows that the ULV spraying neither eliminated nor reduced the number of gravid Aedes mosquitoes. Forty-eight hours after ULV spraying, dead insects, spiders, and even small animals like frogs and snails were found in the ovitrap; in the garden, dead ants and spiders were also observed. Furthermore, one may assume that, following ULV treatment, the destruction of the natural predators of the mosquitoes could have contributed to the increase of immature mosquitoes (Chua et al. 2005). However, it is also known that Ae. aegypti are resistant to pyrethroids-the insecticides typically used in ULV (Leong et al. 2019)—especially in dengue epidemic areas. Other studies have also shown that space-spraying has not been effective in dengue epidemics (Esu et al. 2010). Since Ae. aegypti have been shown to be resistant to pyrethroids in most countries, mosquitoes are given an advantage if managers do not change insecticides.

\section{Vectors of filariasis}

Vectors of filariasis are varied. In Malaysia and the surrounding regions Mansonia mosquitoes are the main vector for Brugian filariasis. As a matter of fact, the first proof of transmission of a human disease by a mosquito was demonstrated by Patrick Manson in South China in 1878 (Mak 1983): Manson demonstrated that Culex quinquefasciatus was responsible for the transmission of Wucheriria bancrofti which had been discovered 15 years earlier. In India and other regions where $W$. bancrofti is predominant $C x$. quinquefasciatus is the vector, whereas Ae. Polynesinsis is the vector in the Pacific islands. Some species of Anopheles are also vectors for filariasis. Thus, one can see that many different species of mosquitoes are involved in the transmission of filariasis. Unlike other vector-borne diseases, a single bite by an infected mosquito will not give rise to filariasis. A male and female microfilaria must be introduced before the person can be infected. In Malaysia, filariasis has been brought to very low levels. The control was based mainly on mass blood surveys and treatment of people rather than directed towards controlling mosquitoes.

\section{Control of Mansonia mosquitoes}

There are six species of Mansonia which have been incriminated as vectors: Ma. annulata, Ma. annulifera, Ma. bonneae, Ma. dives, Ma. indiana and Ma. uniformis (Wharton 1962). Early experiments in the laboratory showed that Mansonia 
were only moderately susceptible to DDT, dieldrin and benzene hexachloride (BHC). Semi-field trials in trap huts against three Mansonia species concluded that dieldrin was more effective than DDT and BHC. A pilot trial was conducted in an area along with mass treatment. There was a decrease in the microfilaria rate but no observable differences in the infection and transmission rates in the vectors (Wharton et al. 1958). This also could be due to animal reservoirs.

The physical removal of floating vegetation is one method of reducing the number of vectors since the immature stages are attached to the roots of plants. Large-scale drainage and irrigation schemes and developments like filling swamps have reduced vector populations. Along with drug administration it was possible to reduce the incidence of filariasis.

\section{Culex and arboviral diseases}

Various species of Culex mosquitoes are vectors of Japanese encephalitis (JE) and West Nile virus. West Nile virus occurs in North America, Africa, Europe, Middle East and West Asia. The Culex mosquitoes obtain the virus while feeding on birds and transmit it to humans. Human-to-human transmission does not take place in West Nile virus, nor in JE. JE is endemic in an area inhabited by about 1.9 billion people in Southeast Asia and Asia, being transmitted by many different species of Culex mosquitoes of which Cx. tritaeniorhynchus and Cx. gelidus are the main vectors (Vythilingam et al. 1997, Kabilan et al. 2004). Fortunately, a vaccine is available for JE. Although a number of JE isolates were found in various species of Culex mosquitoes in a study in Malaysia (Vythilingam et al. 1997), cases of JE are only sporadic and isolated transmissions occur occasionally.

\section{Control of Culex vectors}

To control the vectors of JE, it is recommended to carry out residual spraying of pig farms in endemic areas where the disease is a major problem. In other areas, houses surrounding the pig farms usually have screens on doors and windows to prevent the entry of mosquitoes. In rice-field areas where there is breeding of JE vectors, alternate wet and dry irrigation, or the use of fish or other natural products like Azolla, can be used to control the larvae (Keiser et al. 2005).

Culex quinquefasciatus is a cosmopolitan mosquito found in most parts of the world. It breeds in polluted water such as sewerage drains, cesspits, septic tanks, cesspools. This mosquito plays a major role in lymphatic filariasis; its control is difficult since it has developed insecticide resistance to all four classes of insecticides (Jones et al. 2012). Effective control of Cx. quinquefaciatus is best carried out by thoroughly cleaning the environment, as well as by source reduction and floating polystyrene beads into breeding sites (Jones et al. 2012). 


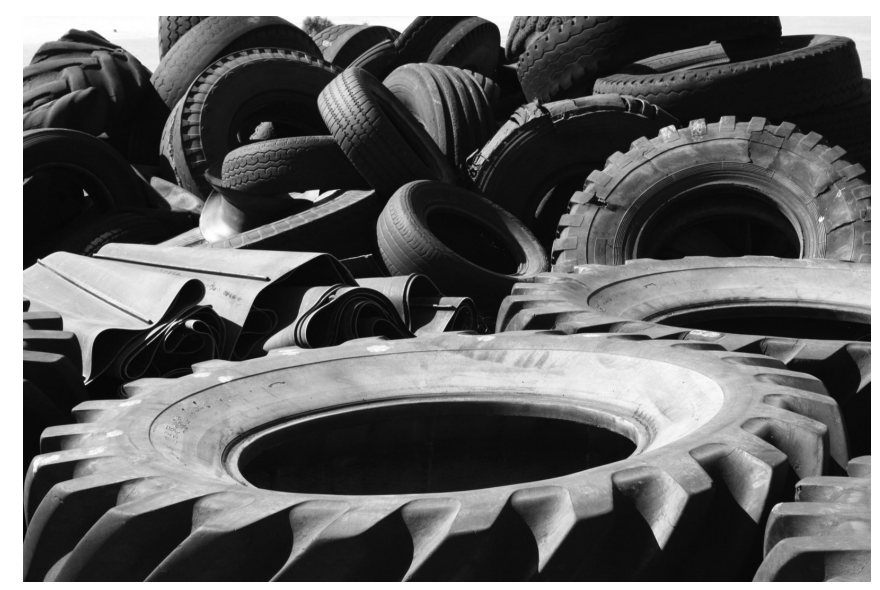

FIGURE 17.2 Discarded tyres near Perth, Australia (Creative Commons; photo by Alex Dawson 2008).

\section{Gene modification}

Mosquitoes do not need passports to cross international borders. In an era in which human agency has reached every corner of the globe and every aspect of life on Earth, humans are responsible for the spreading of mosquitoes, especially the Aedes genus whose eggs can withstand desiccation and are carried across countries and oceans by water trapped in used tyres (Gubler 2012). Accordingly, most of this genus and especially Ae. albopictus has established itself and will probably not be eliminated from the planet in the near future. Control measures are only instituted if mosquitoes are found to be disease bearing (Figure 17.2).

One should also understand that it is not necessary for large numbers of mosquitoes to be present to cause a disease. For example, Ae. aegypti is easily disturbed and thus can feed on multiple hosts to acquire a full blood meal (Scott et al. 1993), meaning that if the mosquito is infected it can pass the pathogen on to many people during a single blood meal (Platt et al. 1997). Singapore is a good example, where the Aedes index is very low but where a large number of cases is reported (Ooi et al. 2006).

Scientists have produced (and are producing) genetically modified (GM) mosquitoes to help control the Ae. aegypti with mixed results. The UK biotech company, Oxitec, has developed a mosquito inserted with a RIDL gene (release of insects carrying a dominant lethal) that can only survive in water with tetracycline. The control strategy is to release the adult males into the wild so that when they mate with the wild females, they will produce offspring that cannot survive, since there is an absence of tetracycline (Massonnet-Bruneel et al. 2013). In Brazil, following large releases of such a mosquito, there are now three different populations of the Ae. aegypti found in nature, with a significant transfer of the tetracycline-dependent genome into the wild population. Eighteen months 
after release of these GM mosquitoes, observations showed that the population which had been initially suppressed began to rebound, becoming as it was before release (Evans et al. 2019). The cautionary note is that these releases may end up promoting more robust populations of mosquitoes in the future, with ultimate effects on the ecosystem remaining unknown.

With the production of the GM mosquitoes, especially the Anopheles, one needs to understand that these mosquitoes are produced in laboratories either in the UK or in USA and they have to be shipped to and released in tropical countries where malaria occurs. The vectors for malaria are varied and many species are involved and the eggs cannot withstand desiccation. This would involve a huge sum of money for a developing country where malaria is occurring and specialized laboratories need to be set up for the production of the GM mosquitoes. Thus, such mosquitoes may only remain as a laboratory tool and are not realistic strategies for public health (Beisel and Boete 2013).

It is also a known fact that mosquitoes have developed resistance to insecticides by changing one or more of their genes (Hemingway et al. 2004) and that malaria parasites have developed resistance to drugs, including artemisinin (Ashley et al. 2014). It is therefore possible that in the long run these GM mosquitoes, which are refractory to the parasites, may one day become susceptible to them. At that point in time, malaria may rebound with a vengeance and the expertise to control the disease and vectors may not be available. We have lessons to learn from what has happened with the COVID-19 pandemic.

Most arthropods and insects are inhabited by Wolbachia bacteria (Moreira et al. 2009). However, Wolbachia is absent in Ae. aegypti. This observation led researchers to inoculate Wolbachia from drosophila into Ae. aegypti. Due to cytoplasmic incompatibility (CI), a male Ae. aegypti with Wolbachia that mates with the wild female will produce inviable progeny, meaning that release of male Ae. aegypti with Wolbachia can suppress this mosquito's population. In addition, a female Ae. aegypti with Wolbachia that mates with a wild male will produce all progeny with Wolbachia, which can be very advantageous for disease management since these bacteria are able to inhibit the ability of Ae. aegypti to carry dengue, chikungunya and Zika viruses (Moreira et al. 2009). As a result, the World Free Mosquito Programme felt it beneficial to release Wolbachia-infected Ae. aegypti in order to infect wild $A$ e. aegypti and make them incapable of spreading viral diseases. What will happen to this initiative in years to come? A recent study showed that Ae. aegypti infected with Wolbachia were still susceptible to chikungunya and Zika viruses at low levels and not entirely refractory as suggested in earlier studies (Tan et al. 2017). Studies also showed that Wolbachia blocked dengue virus in Ae. aegypti by 37.5\% (Bian et al. 2010).

\section{Community participation in vector control}

It is not easy to eliminate mosquitoes from this planet. The common domestic Ae. aegypti was once nearly eliminated from the Americas in the 1930s but then 
rebounded after the programme was stopped (Dick et al. 2012). In theory it looks very simple to get rid of Aedes mosquitoes yet this species has been successful at colonizing new areas and causing more diseases despite human efforts to eradicate it. The typical top-down approach utilized by most governments was not very successful at removing breeding sites. Though successful for a short time, this eradication programme was not sustainable. Thus, it is now generally felt that a bottom-up approach will offer greater success at mosquito control. Community participation has been successful in Cuba, for example, where mosquito control has been more sustainable (Gubler and Clark 1996). Yet in Malaysia, even though community members have demonstrated good knowledge about dengue and its associated mosquitoes, they have shown little willingness to follow through with dengue control (Selvarajoo et al. 2020).

Even if a mosquito does not have the brain of a primate, it has been highly successful at living and sharing the planet with humans. Now researchers are producing GM mosquitoes and releasing them at large scale to outcompete wild mosquitoes. What researchers perhaps fail to appreciate is that GM mosquitoes will eventually die off, allowing wild mosquitoes to bounce back, possibly with a vengeance. Another irony is that we now tell community members that GM mosquitoes are our friends, so please do not kill them. What will happen in the future?

Environmental changes such as land-cover fluxes, deforestation and landscape modifications are also known to affect the distribution and density of mosquitoes. Such changes are the result of human processes. A changing climate can also promote a higher abundance of mosquitoes (Schaffner and Van Bortel 2013) and facilitate their development in a shorter time. It seems that the more humanity modifies the earth, the more that mosquitoes will thrive in our environment.

\section{Conclusion}

Since mosquito control is sometimes dependent on knowing the behaviour of the vectors, mosquitoes not exhibiting such behaviours may not be killed or eliminated. Mosquitoes also have the habit of changing their behaviour in order to escape being killed. When we speak of elimination of malaria or of filariasis, we are referring to eliminating the disease. But the species of mosquitoes responsible for transmitting these diseases will remain; thus the ecosystem will not be disrupted.

In the end, mosquitoes are part of the ecosystem and so will probably never be eliminated. Control measures only target the vector mosquitoes and will allow many other organisms to survive and provide food for still other organisms. The current trend in mosquito control is moving towards creating more mosquitoes, albeit ones that cannot transmit diseases. How far this strategy will succeed remains to be seen. Mosquitoes will not be wiped out from planet Earth. They are smarter than humans and will survive for years to come. To quote Andrew Spielman, "mosquitoes are well adapted to a very unstable, transient 
environment. They're the first organism in and the first out of a newly created body of water" (Spielman 2001). Mosquitoes are here to stay and there is a place for each species. Mosquitoes will thrive in the ecosystem as they have done for millions of years.

\section{Bibliography}

Ashley, E.A., M. Dhorda, R.M. Fairhurst, C. Amaratunga, P. Lim, S. Suon, S. Sreng, J.M. Anderson, S. Mao, B. Sam, and C. Sopha. 2014. Spread of artemisinin resistance in Plasmodium falciparum malaria. New England Journal of Medicine 371(5): 411-423.

Beisel, U., and C. Boëte. 2013. The flying public health tool: Genetically modified mosquitoes and malaria control. Science as Culture 22(1): 38-60.

Bian, G., Y. Xu, P. Lu, Y. Xie, and Z. Xi. 2010. The endosymbiotic bacterium Wolbachia induces resistance to dengue virus in Aedes aegypti. PLoS Pathog 6(4): e1000833.

Chin, W., P.G. Contacos, G.R. Coatney, and H.R. Kimball. 1965. A naturally acquired quotidian-type malaria in man transferable to monkeys. Science 149: 865.

Chua, K., I. Chua, I. Chua, and K. Chua. 2005. Effect of chemical fogging on immature Aedes mosquitoes in natural field conditions. Singapore Medical Journal 46: 639.

Dick, O.B., J.L. San Martín, R.H. Montoya, J. del Diego, B. Zambrano, and G.H. Dayan. 2012. The history of dengue outbreaks in the Americas. American Journal of Tropical Medicine and Hygiene 87: 584-593.

Esu, E., A. Lenhart, L. Smith, and O. Horstick. 2010. Effectiveness of peridomestic space spraying with insecticide on dengue transmission; systematic review. Tropical Medicine E International Health 15: 619-631.

Evans, B.R., P. Kotsakiozi, A.L. Costa-da-Silva, R.S. Ioshino, L. Garziera, M.C. Pedrosa, A. Malavasi, J.F. Virginio, M.L. Capurro, and J.R. Powell. 2019. Transgenic Aedes aegypti mosquitoes transfer genes into a natural population. Scientific Reports 9: 1-6.

Fegan, G.W., A.M. Noor, W.S. Akhwale, S. Cousens, and R.W. Snow. 2007. Effect of expanded insecticide-treated bednet coverage on child survival in rural Kenya: A longitudinal study. Lancet 370: 1035-1039.

Fong, Y.L., F.C. Cadigan, and G.R. Coatney. 1971. A presumptive case of naturally occurring Plasmodium knowlesi malaria in man in Malaysia. Transactions of the Royal Society of Tropical Medicine and Hygiene 65: 839.

Fornace, K.M., N. Alexander, T.R. Abidin, P.M. Brock, T.H. Chua, I. Vythilingam, H.M. Ferguson, B.O. Manin, M.L. Wong, and S.H. Ng. 2019. Local human movement patterns and land use impact exposure to zoonotic malaria in Malaysian Borneo. eLife 8: e47602.

Grignard, L., S. Shah, T.H. Chua, T. William, C.J. Drakeley, and K.M. Fornace. 2019. Natural human infections with Plasmodium cynomolgi and other malaria species in an elimination setting in Sabah, Malaysia. The Journal of Infectious Diseases 220: 1946-1949.

Gubler, D.J. 2001. Human arbovirus infections worldwide. Annals of the New York Academy of Sciences 951: 13-24.

Gubler, D.J. 2012. The economic burden of dengue. American Journal of Tropical Medicine and Hygiene 86: 743-744.

Gubler, D.J., and G.G. Clark. 1996. Community involvement in the control of Aedes aegypti. Acta Tropica 61: 169-179.

Hemingway J, Hawkes NJ, McCarroll L, Ranson H. 2004. The molecular basis of insecticide resistance in mosquitoes. Insect Biochemistry and Molecular Biology 34(7):653-665. 
Hii, J., and L. Rueda. 2013. Malaria vectors in the Greater Mekong Subregion: Overview of malaria vectors and remaining challenges. Southeast Asian Journal of Tropical Medicine and Public Health 44(Supplement 1): 73-165.

Hussin, N., Y.A.L. Lim, P.P. Goh, T. William, J. Jelip, and R.N. Mudin. 2020. Updates on malaria incidence and profile in Malaysia from 2013 to 2017. Malaria Journal 19: 55.

Imwong, M., W. Madmanee, K. Suwannasin, C. Kunasol, T.J. Peto, R. Tripura, L. von Seidlein, C. Nguon, C. Davoeung, and N.P. Day. 2019. Asymptomatic natural human infections with the simian malaria parasites Plasmodium cynomolgi and Plasmodium knowlesi. The Journal of Infectious Diseases 219: 695-702.

Jones, C.M., C. Machin, K. Mohammed, S. Majambere, A.S. Ali, B.O. Khatib, J. Mcha, H. Ranson, and L.A. Kelly-Hope. 2012. Insecticide resistance in Culex quinquefasciatus from Zanzibar: Implications for vector control programmes. Parasites \& Vectors 5: 78.

Kabilan, L., R. Rajendran, N. Arunachalam, S. Ramesh, S. Srinivasan, P.P. Samuel, and A. Dash. 2004. Japanese encephalitis in India: An overview. Indian Journal of Pediatrics 71: 609-615.

Keiser, J., M.F. Maltese, T.E. Erlanger, R. Bos, M. Tanner, B.H. Singer, and J. Utzinger. 2005. Effect of irrigated rice agriculture on Japanese encephalitis, including challenges and opportunities for integrated vector management. Acta Tropica 95: 40-57.

Leong, C.-S., I. Vythilingam, J.W.-K. Liew, M.-L. Wong, W.S. Wan-Yusoff, and Y.L. Lau. 2019. Enzymatic and molecular characterization of insecticide resistance mechanisms in field populations of Aedes aegypti from Selangor, Malaysia. Parasites \& Vectors 12: 236.

Mak, J. 1983. Filariasis. Kuala Lumpur: Institute for Medical Research, p. 108.

Massonnet-Bruneel, B., N. Corre-Catelin, R. Lacroix, R.S. Lees, K.P. Hoang, D. Nimmo, L. Alphey, and P. Reiter. 2013. Fitness of transgenic mosquito Aedes aegypti males carrying a dominant lethal genetic system. Plos One 8: e62711.

Moorhouse, D.E. 1965. Some entomological aspects of the malaria eradication pilot project in Malaya. Journal of Medical Entomology 2: 109-119.

Moreira, L.A., I. Iturbe-Ormaetxe, J.A. Jeffery, G. Lu, A.T. Pyke, L.M. Hedges, B.C. Rocha, S. Hall-Mendelin, A. Day, and M. Riegler. 2009. A Wolbachia symbiont in Aedes aegypti limits infection with dengue, chikungunya, and Plasmodium. Cell 139: 1268-1278.

Mouchet, J. 1994. DDT and public health. Cahiers d'études et de recherches francophones / Santé 4: 257-262.

Mudin, R.N. 2015. Dengue incidence and the prevention and control programme in Malaysia. International Medical Journal Malaysia 14: 5-9.

Nam, V.S., N.T. Yen, H.M. Duc, T.C. Tu, V.T. Thang, N.H. Le, L. Le Loan, V.T.Q. Huong, L.H.K. Khanh, and H.T.T. Trang. 2012. Community-based control of Aedes aegypti by using Mesocyclops in southern Vietnam. American Journal of Tropical Medicine and Hygiene 86: 850-859.

Ooi, E.-E., K.-T. Goh, and D.J. Gubler. 2006. Dengue prevention and 35 years of vector control in Singapore. Emerging Infectious Diseases 12: 887.

Platt, K.B., K.J. Linthicum, K. Myint, B.L. Innis, K. Lerdthusnee, and D.W. Vaughn. 1997. Impact of dengue virus infection on feeding behavior of Aedes aegypti. American Journal of Tropical Medicine and Hygiene 57: 119-125.

Schaffner F and Van Bortel AW. 2013. Public health significance of invasive mosquitoes in Europe. Clinical Microbiology and Infection 19: 685-692.

Scott, T.W., E. Chow, D. Strickman, P. Kittayapong, R.A. Wirtz, L.H. Lorenz, and J.D. Edman. 1993. Blood-feeding patterns of Aedes aegypti (Diptera: Culicidae) collected in a rural Thai village. Journal of Medical Entomology 30: 922-927. 
Selvarajoo, S., J.W.K. Liew, W. Tan, X.Y. Lim, W.F. Refai, R.A. Zaki, N. Sethi, W.Y.W. Sulaiman, Y.A.L. Lim, and J. Vadivelu. 2020. Knowledge, attitude and practice on dengue prevention and dengue seroprevalence in a dengue hotspot in Malaysia: A cross-sectional study. Scientific Reports 10: 1-13.

Singh, B., K. Kadir, T. Hu, T. Raja, D. Mohamad, L. Lin, and K. Hii. 2018. Naturally acquired human infections with the simian malaria parasite, Plasmodium cynomolgi, in Sarawak, Malaysian Borneo. International Journal of Infectious Diseases 73: 68.

Singh, B., L.K. Sung, A. Matusop, A. Radhakrishnan, S.S.G. Shamsul, J. Cox-Singh, A. Thomas, and D.J. Conway. 2004. A large focus of naturally acquired Plasmodium knowlesi infections in human beings. Lancet 363: 1017-1024.

Singh, J., A.S. Tham, and WHO. 1988. Case History on Malaria Vector Control Through the Application of Environmental Management in Malaysia. Geneva: World Health Organization.

Solomon, T. 2006. Control of Japanese encephalitis-within our grasp? New England Journal of Medicine 355: 869.

Spielman, A. 2001. Mosquito: A Natural History of Our Most Persistent and Deadly Foe. New York: Hyperion.

Ta, T.H., S. Hisam, M. Lanza, A.I. Jiram, N. Ismail, and J.M. Rubio. 2014. First case of a naturally acquired human infection with Plasmodium cynomolgi. Malaria Journal 13: 1-7.

Tan, C.H., P.J. Wong, M.I. Li, H. Yang, L.C. Ng, and S.L. O’Neill. 2017. wMel limits zika and chikungunya virus infection in a Singapore Wolbachia-introgressed Ae. aegypti strain, wMel-Sg. PLoS Neglected Tropical Diseases 11: e0005496.

Vythilingam, I., K. Oda, S. Mahadevan, G. Abdullah, C.S. Thim, C.C. Hong, B. Vijayamalar, M. Sinniah, and A. Igarashi. 1997. Abundance, parity, and Japanese encephalitis virus infection of mosquitoes (Diptera: Culicidae) in Sepang District, Malaysia. Journal of Medical Entomology 34: 257-262.

Vythilingam, I., and W. Wan-Yusoff. 2017. Dengue vector control in Malaysia: Are we moving in the right direction. Tropical Biomedicine 34: 746-758.

Vythilingam, I., M. Wong, and W. Wan-Yussof. 2018. Current status of Plasmodium knowlesi vectors: A public health concern? Parasitol 145: 32-40.

Warren, M.W., W.H. Cheong, H.K. Fredericks, and G.R. Coatney. 1970. Cycles of jungle malaria in West Malaysia. American Journal of Tropical Medicine and Hygiene 19: 383.

Warren, M.W., and R.H. Wharton. 1963. The vectors of simian malaria: Identity, biology, and geographical distribution. Journal of Parasitolology 49: 892-904.

Watson, M. 1921. The Prevention of Malaria in the Federated Malay States: A Record of Twenty Year's Progress. New York: E.P. Dutton \& Company.

Wharton, R. 1960. Studies on filariasis in Malaya: Field and laboratory investigations of the vectors of a rural strain of Wuchereria bancrofti. Annals of Tropical Medicine and Parasitology 54: 78-91.

Wharton, R., J. Edeson, T. Wilson, and J. Reid. 1958. Studies on filariasis in Malaya: Pilot experiments in the control of filariasis due to Wuchereria malayi in East Pahang. Annals of Tropical Medicine E Parasitology 52: 191-205.

Wharton, R.H. 1962. The biology of Mansonia mosquitoes in relation to transmission of filariasis in Malaya. Bulletin of the Institute for Medical Research, Malaysia 11: 1-114.

World Health Organization. 2017. A Framework for Malaria Elimination. Geneva: World Health Organization.

World Health Organization. 2019. World Malaria Report 2019. Reference Source. 


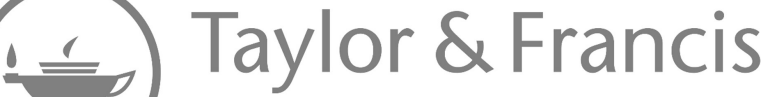

Taylor \& Francis Group

http://taylorandfrancis.com 